\title{
Posible relación de loratadina con el desenlace fatal en un paciente de riesgo
}

\author{
Emilio Pol Yanguas', Rita Mirch², Enrique Mayoral Borrell², Paco Martínez Granados ${ }^{3}$
}

1. Doctor en Farmacia, Máster en Medicina Humanitaria. Especialista en Farmacia de Hospital. Centro Dr. Esquerdo, servicio de Farmacia. San Juan, Alicante. 2. Estudiantes de Farmacia. Universidad Miguel Hernández. 3. Máster en Neuropsicofarmacología y Toxicomanías. Farmacéutico especialista de área. Hospital General Universitario. San Juan, Alicante.

\section{PALABRAS CLAVE}

Loratadina, arritmia ventricular, muerte, enfermos mentales, efecto mariposa

\author{
ABREVIATURAS \\ EPOC: enfermedad pulmonar \\ obstructiva crónica \\ COPD: chronic obstructive \\ pulmonary disease \\ UCI: unidad de cuidados \\ intensivos \\ DDD: dosis diaria definida
}

\section{KEYWORDS}

Loratadine, ventricular arrhythmias, death, mental illness, butterfly effect

\section{RESUMEN}

Un varón de 62 años, polimedicado de forma crónica por enfermedad mental grave y persistente, EPOC, diabetes e hipotiroidismo, estabilizado desde hace bastantes meses, inició un tratamiento con loratadina por rinitis. Al día siguiente es encontrado desvanecido sin conocimiento y trasladado de urgencia al hospital general para cuidados intensivos, donde fallece un día después. El análisis del caso revela un posible "efecto mariposa" con desenlace fatal, desencadenado por un fármaco de bajo riesgo, dada la situación "al límite" de la terapia previa.

Possible link of loratadine with a fatal outcome in an at-risk patient

\section{ABSTRACT}

A 69-year-old man, who has been clinically chronic for severe and persistent mental illness, COPD, diabetes and hypothyroidism, stabilized for several months, began treatment with loratadine for rhinitis. The next day he is found unconscious and rushed to the general hospital for intensive care, where he dies a day later. The case analysis reveals a possible "butterfly effect," a fatal outcome caused by a lowrisk drug, given the "borderline" situation of prior therapy.

\section{Introducción}

Los pacientes psiquiátricos tienen un elevado riesgo de sufrir interacciones adversas, dada la polifarmacia que reciben. Presentamos un caso de posible "efecto mariposa". La adición de loratadina (en principio con bajo riesgo y por ello de posible adquisición sin receta médica [1]) a un tratamiento intenso, que coloca al paciente en una situación "metaestable", fue el posible desencadenante de una reacción adversa grave: desvanecimiento y posterior muerte del paciente.

\section{Presentación del caso}

Paciente de 62 años, ingresado de forma involuntaria en un centro para rehabilitación de enfermos mentales. Desde 1994 ha sufrido varios ingresos, hasta el último que duraba desde 2013. Durante su estancia fue tratado con diversos fármacos dependiendo de su estado y progreso de las patologías. El tratamiento del paciente se había mantenido estable desde 2015, hasta que debido a un episodio de rinitis se añadió loratadina al tratamiento habitual (tabla 1 ). 
Tabla 1 Tratamiento farmacológico del paciente en el momento del incidente. Datos obtenidos de las fichas técnicas [1]. DDD obtenidas de la web [2]

\begin{tabular}{|c|c|c|c|c|c|c|}
\hline Medicación & Pauta & Indicación & Dosis & $\begin{array}{c}\text { Dosis } \\
\text { mantenimiento }\end{array}$ & Contraindicaciones & RAM $^{*}$ \\
\hline $\begin{array}{l}\text { Clonazepam } \\
0,5 \mathrm{mg}\end{array}$ & $\begin{array}{l}\text { 1-1-1-1 } \\
\text { crónico }\end{array}$ & Epilepsia & $\begin{array}{l}2 \mathrm{mg}= \\
0,25 \mathrm{DDD} \\
\text { (para epilepsia) }\end{array}$ & & $\begin{array}{l}\text { Dependencia } \\
\text { alcohólica } \\
\text { Insuficiencia } \\
\text { respiratoria grave }\end{array}$ & $\begin{array}{l}\text { Agresividad } \\
\text { Insuficiencia cardiaca, } \\
\text { incluida parada cardiaca }\end{array}$ \\
\hline $\begin{array}{l}\text { Fluticasonal } \\
\text { salmeterol } \\
250 / 25 \mathrm{mcg}\end{array}$ & $\begin{array}{l}\text { 1-0-1-0 } \\
\text { crónico }\end{array}$ & Asma & 2 inhalaciones & $\begin{array}{l}2 \mathrm{inh} / 2 \text { veces } \\
\text { al dia }\end{array}$ & & $\begin{array}{l}\text { Disnea } \\
\text { Trastorno } \\
\text { hidroelectrolítico } \\
\text { Hiperglucemia }\end{array}$ \\
\hline $\begin{array}{l}\text { Haloperidol } \\
5 \mathrm{mg}\end{array}$ & $\begin{array}{l}\text { 1-1-1-0 } \\
\text { crónico }\end{array}$ & Neuroléptico & $\begin{array}{l}15 \mathrm{mg}=1,9 \\
\mathrm{DDD}\end{array}$ & $\begin{array}{l}10-20 \mathrm{mg} / 3 \\
\text { veces al dia }\end{array}$ & $\begin{array}{l}\text { Depresión del SNC } \\
\text { producida por el } \\
\text { alcohol u otros } \\
\text { medicamentos } \\
\text { depresores }\end{array}$ & $\begin{array}{l}\text { Trastornos oculares } \\
\text { Hiperprolactinemia }\end{array}$ \\
\hline $\begin{array}{l}\text { Levotiroxina } \\
50 \mathrm{mcg}\end{array}$ & $\begin{array}{l}\text { 3-0-0-0 } \\
\text { crónico }\end{array}$ & Hipotiroidismo & $\begin{array}{l}150 \mathrm{mcg}=1 \\
\mathrm{DDD}\end{array}$ & $100-200 \mathrm{mg}$ & & Agitación \\
\hline $\begin{array}{l}\text { Loratadina } \\
10 \mathrm{mg}\end{array}$ & $\begin{array}{l}1-0-0-0 \\
5 \text { dias }\end{array}$ & Rinitis alérgica & $\begin{array}{l}10 \mathrm{mg}=1 \\
\mathrm{DDD}\end{array}$ & 10 mg/día & & \\
\hline $\begin{array}{l}\text { Metformina } \\
850 \mathrm{mg}\end{array}$ & $\begin{array}{l}\text { 0-1-1-0 } \\
\text { crónico }\end{array}$ & $\begin{array}{l}\text { Diabetes } \\
\text { mellitus tipo } 2\end{array}$ & $\begin{array}{l}1.700 \mathrm{mg}= \\
0,9 \mathrm{DDD}\end{array}$ & Dmax $=3 \mathrm{~g} / \mathrm{di} a$ & $\begin{array}{l}\text { Alcoholismo } \\
\text { Insuf. respiratoria }\end{array}$ & \\
\hline $\begin{array}{l}\text { Quetiapina } \\
400 \mathrm{mg}\end{array}$ & $\begin{array}{l}\text { 1-1-1-0 } \\
\text { crónico }\end{array}$ & $\begin{array}{l}\text { Esquizofrenia, } \\
\text { TDM }\end{array}$ & $\begin{array}{l}1200 \mathrm{mg}=3 \\
\mathrm{DDD}\end{array}$ & $\begin{array}{l}400 \text { a } 800 \mathrm{mg} / \\
\text { dia }\end{array}$ & & $\begin{array}{l}\text { Diabetes mellitus } \\
\text { Hipotiroidismo } \\
\text { Picores }\end{array}$ \\
\hline $\begin{array}{l}\text { Tiotropio } \\
2,5 \text { mcg/ } \\
\text { puls. }\end{array}$ & $1-0-0-0$ & EPOC & 2 pulsaciones & $\begin{array}{l}5 \text { mcg en dos } \\
\text { pulsaciones/dia }\end{array}$ & & Trastornos oculares \\
\hline $\begin{array}{l}\text { Valproico ac. } \\
500 \mathrm{mg}\end{array}$ & $\begin{array}{l}\text { 1-1-1-0 } \\
\text { crónico }\end{array}$ & $\begin{array}{l}\text { Epilepsia } \\
\text { Manía }\end{array}$ & $\begin{array}{l}1500 \mathrm{mg}=1 \\
\mathrm{DDD}\end{array}$ & $15-20 \mathrm{mg} / \mathrm{kg}$ & & $\begin{array}{l}\text { Agresividad } \\
\text { Trastorno } \\
\text { hidroelectrolítico }\end{array}$ \\
\hline
\end{tabular}

* Efectos descritos en la ficha técnica observados en el paciente.

Al día siguiente es encontrado inconsciente en uno de los lugares de paseo y es trasladado al hospital general, ingresando en la UCI en estado comatoso. Un día más tarde, el paciente fallece por fallo cardíaco debido a fibrilación ventricular.

En la historia constaban los siguientes diagnósticos psiquiátricos: esquizofrenia paranoide, trastorno por dependencia de alcohol y conducta agresiva. También constaban otros problemas de salud: hipotiroidismo, diabetes mellitus tipo 2, enfermedad pulmonar obstructiva crónica (EPOC) y tabaquismo. Había sido intervenido por cataratas y por lipomas.

\section{Análisis y evaluación}

Cabe preguntarse por la relación entre el evento adverso y el trata- miento que el paciente recibe. Señalamos la duplicidad del tratamiento antipsicótico (haloperidol y quetiapina), a dosis elevadas, y el uso de otros fármacos con indicaciones diferentes a los diagnósticos listados en el paciente (ácido valproico), incluso contraindicados (clonazepam) (tabla 1). La dosis máxima recomendada de quetiapina es $800 \mathrm{mg} /$ día. La dosis diaria antipsicótica total es de 5 DDD [2]. El tratamiento presenta hasta siete posibles interacciones [3], destacando las siguientes:

- Torsade de pointes y muerte súbita: haloperidol puede causar una prolongación del intervalo QT relacionada con la dosis. La coadministración con otros agentes que pueden prolongar el intervalo QT (quetiapina y fluticasona/salmeterol) puede resultar en efectos aditi- vos y un mayor riesgo de arritmias ventriculares, incluyendo torsade de pointes y muerte súbita.

- Taquicardia: la interacción entre haloperidol y/o quetiapina, y tiotropio puede producir efectos anticolinérgicos aditivos como taquicardia. Los agentes con propiedades anticolinérgicas centrales (por ejemplo, loratadina, quetiapina y haloperidol) pueden tener efectos adyuvantes parasimpaticolíticos y depresores del sistema nervioso central cuando se usan en combinación. También asociados a muerte súbita.

Otras interacciones [3] son consecuencia de la cascada de medicación generada por el tratamiento de problemas de salud, provocados a su vez por los fármacos psiquiátricos. Entre 
ellas, la reducción de la efectividad de metformina por quetiapina y levotiroxina.

El número máximo recomendado de medicamentos en la categoría de "fármacos del sistema nervioso central" que se toman simultáneamente no debe ser superior a tres [3]. El paciente recibía cuatro (haloperidol, quetiapina, clonazepam y valproico). Se recomienda no tomar simultáneamente más de un fármaco antipsicótico. Asimismo, se emplean dosis antipsicóticas elevadas, no se recomienda más de 2 DDD en mantenimiento [4]. Clonazepam está clasificado como anticonvulsivante y la correspondiente DDD se refiere a esta indicación. El paciente recibe $2 \mathrm{mg}$ de clonazepam, 0,25 DDD [2]. Pero este no tiene diagnóstico de convulsiones y lo usa como psicofármaco. Como ansiolítico [5], $2 \mathrm{mg} /$ día de clonazepam equivalen a $40 \mathrm{mg} /$ día de diazepam (4 DDD) [2]. El uso crónico de benzodiacepinas no está indicado en las psicosis, además la depresión central que causan aumentan las dificultades respiratorias de la EPOC.

La introducción de loratadina al tratamiento el día anterior al suceso pudo actuar como "gota que colma el vaso", desencadenante del mismo, no por los efectos del fármaco en sí, sino porque este paciente se encontraba en un delicado equilibro con todos los componentes de su tratamiento, "el aleteo de la mariposa que desencadena la catástrofe”. Entre los efectos adversos de loratadina en ficha técnica se incluyen "taquicardia y palpitaciones", aunque con frecuencia muy rara (1).

Empleando el algoritmo de Karch-Lasagna [6] (tabla 2), la relación de causalidad planteada es "posible", ya que cumple los requisitos de secuencia temporal (ha coincidido la introducción del fármaco con el incidente), de ser una respuesta conocida y de no encontrar una explicación alternativa (de haber sido a causa directa de otros fármacos podría haber sucedido durante los 2 años que llevaba el mismo tratamiento).

Loratadina es un antihistamínico usado frecuentemente para patologías comunes, como rinitis alérgica o reacciones cutáneas. Se trata un medicamento con algunas presentaciones farmacéuticas de venta sin prescripción médica [1], lo que hace que el caso presentado sea especialmente relevante desde la perspectiva

Tabla 2 Ejecución del algoritmo de Karch-Lasagna

\begin{tabular}{|l|c|c|c|c|}
\hline \multirow{2}{*}{ Criterio } & \multicolumn{4}{|c|}{ Valoración de la relación causal } \\
\cline { 2 - 5 } & Definida & Probable & Posible & Condicional \\
\hline Secuencia temporal & Si & Si & Si & Si \\
\hline $\begin{array}{l}\text { Respuesta al fármaco } \\
\text { conocida }\end{array}$ & Si & Si & Si & No \\
\hline $\begin{array}{l}\text { Presencia de una explicación } \\
\text { alternativa para la reacción }\end{array}$ & No & No & Si & No \\
\hline $\begin{array}{l}\text { Mejora al retirar el } \\
\text { medicamento }\end{array}$ & Si & Si & Si o No & Si o No \\
\hline Reaparece al reintroducirlo & Si & ¿? & ¿? & ¿? \\
\hline
\end{tabular}

de la atención farmacéutica. El caso fue notificado a farmacovigilancia. La experiencia indica que loratadina es un medicamento muy seguro, y su contribución al desenlace descrito es comparable al de "un eco que desencadena un alud en la montaña".

\section{Intervención/resultado}

No hubo opción a intervención por parte del farmacéutico ya que el proceso de prescripción-dispensación-administración se interrumpió con el traslado y dada la rapidez del desenlace fatal. No obstante, los respectivos farmacéuticos efectuaron con los equipos asistenciales, tanto en el centro residencial psiquiátrico como en la UCI del hospital general, un análisis de la implicación de la farmacoterapia en la génesis del problema y se remitió al centro regional de farmacovigilancia junto con la preceptiva tarjeta amarilla. El caso presentado era un candidato claro a la desprescripción. En los pacientes psiquiátricos crónicos polimedicados se efectúa rutinariamente una revisión de tratamiento mensualmente y con cada cambio, y el farmacéutico emite una nota de aviso cuando detecta el uso de dosis elevadas o la presencia de potenciales interacciones o situaciones de contraindicación, entre otras intervenciones. Desgraciadamente aunque estos problemas potenciales de seguridad relacionados con los medicamentos son extraordinariamente frecuentes en los pacientes psiquiátricos, y es una de las más probables causas del exceso de mortalidad de esta población [7], el farmacéutico sigue sin ser incorporado en las políticas de salud mental.

\section{Referencias bibliográficas}

1. Agencia Española del Medicamento y Productos Sanitarios (AEMPS) [Internet]. Centro de información online de medicamentos de la AEMPS. [Acceso 30/3/2017]. Disponible en: https://www.aemps.gob.es/cima/publico/ home.html

2. WHO Collaborating Center for Drug Statistics Methodology [Internet]. ATC/ DDD Index-2017. [Acceso 30/3/2017]. Disponible en: https://www.whocc.no/ atc_ddd_index/

3. Drugs.com [Internet]. Drug Interactions Checker. [Acceso 30/3/2017]. Disponible en: https://www.drugs.com/drug_ interactions.php

4. Buchanan RW. Kreyenbuhl J, Kelly DL, Noel JM, Boggs DL, Fischer BA, et al. Schizofrenia Patient Outcomes Team (PORT). The 2009 schizophrenia PORT psychopharmacological treatment recommendation and summary statements. Schizophr Bull. 2010; 36:71-93. doi:10.1093/schbul/sbp 116

5. Marangell L, Yudofsky S, Silver J. Psicofarmacología y terapia electroconvulsiva. En: Hales R, Yudofsky S, Talbott J. DSM-IV Tratado de psiquiatría. 3a Edición. Barcelona: Masson; 2000. 1027-1131.

6. Laporte JR, Tognoni G [Internet]. Principios de epidemiologia del medicamento. [Acceso 30/3/2017]. Disponible en: http://www.icf.uab.cat/assets/pdf/ productes/libres/pem.pdf

7. Liu NH, Daumit GL, Dua T, Aquila R, Charlson F, Cuijpers P, et al [Internet]. Mortalidad excesiva en personas con trastornos mentales graves: un modelo de intervención multinivel y prioridades para el ejercicio clínico, las políticas y los programas de investigación.World Psychiatry (edición en Español), 2017; 15:30-40. [Acceso 19/4/17]. Disponible en: http://www. wpanet.org/uploads/Publications/ WPA_Journals/World_Psychiatry/ Past_Issues/Spanish/WPA_SPANISH_1_2017.pdf 